\title{
una narrazione
}

Un museo stabilizza, pro tempore, una visione delle cose. Nasce da conflitti, visibili o fra le quinte, e ne cristallizza l'esito, per tanto o per poco. Specie in certi ambiti, non c'entra necessariamente con la verità, non in senso stretto. Vedi gli itinerari e gli arrangiamenti in corsa nella rete pur geograficamente disomogenea dei musei del Risorgimento, quegli apparati pubblici per la memoria che non si è stati invece in grado di costruire dopo la Seconda guerra mondiale, in presenza di un "Secondo Risorgimento" ancora più contrastato del primo. Nascono quando Mazzini non ha ancora trovato un suo ruolo riconosciuto in piazza e a scuola. Vengono aggiornati in coda, prima con la Grande guerra, poi con i "caduti della rivoluzione fascista", poi con l'Impero; e attorno al centenario dell'Unità nel 1961 - almeno a Torino - con le bandiere rosse a suo tempo predate dagli squadristi ed esibite come cimeli, a rovescio, nella Mostra del decennale, per una proposta interpretativa della Resistenza e del movimento operaio che si intreccia a una interpretazione del Risorgimento.

Pensiero forte, comunque, abbastanza forte da imporre una visione, questa, appunto, cristallizzazione provvisoria degli eventi trascorsi: eventi fondativi. Oggi non ci si riuscirebbe. I Borboni appaiono miti fantoccetti quando dolcemente insinuano in tv i pregi del caffè intitolato alla dinastia, ma sono già da tempo assai meno miti, nelle contese revisioniste del 2011 ecc.; e contribuirebbero a impedire la nascita di un equilibrio come quello che ha trovato forma tra fine Ottocento e inizio Novecento: in alleanza di fatto con altri riaffioramenti di passati dismessi e riattivati. A Padova, dopo una parentesi di trent'anni, si è riusciti nel 2004 a 
riaprire e rinnovare il Museo del Risorgimento e dell'età contemporanea nelle confacenti sale superiori del Caffè Pedrocchi, monumento risorgimentale in se stesso. Niente da fare a Venezia, nonostante reiterati tentativi e larvate promesse. A Venezia - con il 1848-49 di cui è stata capace - un Museo del Risorgimento, che c'era e molto ricco e variegato, non c'è più da trent'anni: prima ne hanno prelevato le sale in nome di mostre d'arte; poi, chiuse le mostre, il materiale è rimasto comunque confinato in depositi e casse; e alla fine le ex-sale del Museo del Risorgimento sono diventate la sale dell'Imperatrice Sissi - la consorte di Francesco Giuseppe! -, che a differenza di Manin ha trovato finanziamenti per il restauro, energie e cure per sorgere, e pubblico pagante al botteghino. È un segno del mercato, ma anche dei tempi. E del fatto che gli apparati museali sono il frutto di circostanze e concause: che a Venezia abbia fatto massa critica l'enorme patrimonio artistico portando e mantenendo al vertice dei Musei civici illustri storici e critici dell'arte non può certo considerarsi un caso né un fatto di per sé negativo, ma questo ha contribuito a definire delle priorità, che non contemplano ora un conflitto con le autorità leghiste e paraleghiste su un fronte - quello della storia politica - considerato ostile o secondario. Strategie interne agli apparati culturali che colludono con le strategie esterne degli apparati politici, e con lo spirito dei tempi.

A Predappio - a me pare - potrebbe forse servire, a stemperare lo scontro e indicare una possibile via d'uscita, una mossa del cavallo. Basta dividersi su "museo sì / museo no", e se riferirlo al fascismo, al Novecento, chiamarlo museo o centro o diversamente. Predappio è Predappio, l'oggetto da lasciare al centro è Predappio stessa, una località tutta e irriducibilmente sagomata e impregnata di una certa storia: è stata fra le due guerre costruita e contrassegnata come un luogo della memoria nella storia d'Italia, e su questo non ci piove: dei nostalgici, con soggettività diverse, ma più oggettivamente di tutti. Questo è ormai il destino dei luoghi, per un itinerario complessivo, dalla nascita alla morte, che investe Mussolini e tutta una genealogia familiare. La collocazione del punto di osservazione nell'ex Casa del fascio, quindi, è adeguata, e del tipo di quelle adottate molte volte in Germania, così spesso ricordata come modello, per incistare la memoria ragionante dei luoghi nei luoghi, negli ambienti, negli edifici stessi di personaggi ed eventi. La casa di nascita, la Casa del fascio, gli edifici marcati da una certa architettura, e la maestra, il fabbro, tutto ciò che fu per decenni e per milioni di persone - ci piaccia o no - una concrezione materiale e simbolica, il repertorio di una religione civile, può essere visitato oggi con atteggiamento conoscitivo, e non necessariamente adorante. Inserendo, così, fra i pellegrinaggi alla tomba e il commercio di cimeli nazifascisti, da una parte, e la tradizione amministrativa di 
sinistra della cittadina, dall'altra, un accompagnamento alla lettura dei luoghi la più seria, documentata, filologica, "raffreddata" possibile.

Non vedo ora come necessario esplicitare il passaggio successivo, ma ritengo corretto da parte mia farlo lo stesso, correndo sia pure il rischio di fraintendimenti. Personalmente, ritengo che una giusta dose di pietas contemplativa e pensosa non guasterebbe il nostro antifascismo: non stiamo parlando solo di loro, stiamo parlando di noi, o dei nostri padri e madri, delle matrici plurime del Fascio, delle ambivalenze dissociative, ma iper-italiane, della Romagna rosso-nera; e, per ineludibile sottinteso, anche di quello che era stato, prima, il Duce, il direttore dell'“Avanti!", il leader del futuro anche per i prossimi "ordinovisti”, uno che di romagnoli e di uomini e donne di sinistra se ne porta dietro tanti, quando rovescia le previsioni e pretende di fare la "rivoluzione" attraverso la guerra e in nome della nazione. Ora, io non penso che proprio qui, in un paesaggio così fortemente connotato, il museo "generale" dell'Italia fascista, fatto eventualmente uscire dalla porta, possa e debba rientrare dalla finestra; però a Predappio c'è un concentrato di simboli che è stato costruito rielaborando e caricando di significati personaggi e circostanze fattuali, che vanno raccolti e messi a frutto, e non dispersi. Succede dovunque - in un paese come il nostro, dalla stratigrafia storica tanto complessa - di aggirarci, coscienti o meno, in un palinsesto, fatto anche di reperti nati come autobiografie di epoche e personaggi. Chiaro che, nel caso del fascismo, il passato risulta tuttora più contendibile, se lo rimane anche per passati che si potevano ritenere spenti. È ciò che complica il rapporto e anima questo dibattito. La retorica, la narrazione e la didattica militante di allora possono funzionare come lucida rappresentazione a posteriori di un itinerario di fede collettiva? Quella fede non c'è più, ma c'è stata per molti, e poiché da molto tempo non ce la caviamo più relegando il fascismo a "parentesi" - prodotto da invasioni di barbari venute da fuori - Predappio appare un luogo appropriato per analizzarne la messa in forma: narrazione di una narrazione. Che Predappio sia stata a lungo rossa nel dopoguerra e tuttora sia rosa - o quello che è - non esime, e forse anzi incoraggia e giustifica, la possibilità di un orizzonte biografico. Questione di approccio, naturalmente, di saperlo fare. Non dico sia facile.

Problema maggiore, la tomba. Ormai c'è, e ci sarà. È il punto più arduo da affrontare nel percorso conoscitivo, poiché a tutt'oggi la si visita anche - impossibile scordarlo, ma impossibile anche evitarlo - con intenti apologetici. Silentium. Qui deve scattare - se ci si riesce - qualche cosa di alto e dignitoso, la zona di rispetto che conclude anche l'atto di giustizia nei confronti dei condannati a morte. Per tutta la vita, Mussolini - una volta passato il guado - si è visto preconizzare, dai suoi stessi amici, una fine simile a quella di Cola di Rienzo; e lui stesso si è arro- 
vellato, ha pensato e scritto, sulla fine dei Cesari e dei tiranni all'incontro fatale con i tirannicidi. Questa messa a morte c'è stata: condanna, fucilazione, ostensione del corpo sulla pubblica piazza; e poi anni di occultamento del cadavere, sino all'arrivo al cimitero di Predappio. È tutto. Tante altre cose e responsabilità non si cancellano, ma quanto meno in rapporto a questo suo capo - tanto amato e odiato - il popolo italiano non rimane in credito. E, con maggiore o minor coscienza di sé - portatore com'è di una storia grande e drammatica - può ridiventare nel suo insieme, e nelle sue diverse componenti, antifascista e fascista, il popolo dei Sepolcri di Ugo Foscolo e persino, almeno nei suoi meccanismi generativi, dell'Inno di Garibaldi.

Se posso fare un riferimento personale, mi rivedo nella cripta - quell'unica volta che negli anni Novanta ci sono andato, in vista dei Luoghi della memoria, che poi non me la sono sentita di fare io stesso, Predappio, appunto, e ho chiesto a Massimo Baioni. Stavo in un canto, ben distinto e per conto mio, e assistevo al reiterato arrivo di questi gruppi e famiglie, devoti o curiosi, va' a sapere; e vedevo perché i registri potevano riempirsi presto di firme e si succedevano l'uno all'altro (come la volta che, poco meno imbarazzato, ero stato in visita fra i pellegrini alla tomba del beato Leopoldo Mandic, nella chiesa dei Cappuccini a Padova). Sarebbe fuori luogo ipotizzare che un domani, non si sa fra quanto e quanto a lungo, possano essere questi, più o meno, al termine dell'itinerario predappiese - nel punto, il mcd più divisivo e dolente - la dialettica delle presenze e dei sentimenti, e il gioco delle parti? 